\title{
Study on Approaches to Personal Development of Young University Teachers
}

\author{
Meng Xiaoli ${ }^{1}$, Guo Junjie ${ }^{2}$ \\ ${ }^{1}$ Shandong Jiaotong University, Weihai, China \\ ${ }^{2}$ Shandong Jiaotong University, Weihai, China \\ 1Xiaoli198309@163.com; ㄹojie920@sina.com
}

Key Words: University young teachers, personal development, sense of self-worth, growth mindset, emotion management

\begin{abstract}
As major force of university development, young university teachers attract extensive attention. Many researchers carried out study on analysis of their psychological status, and further discussed what universities could do to facilitate young teachers' psychological development. However, this paper focuses on self-development of young university teachers and emphasizes the active role they can play in personal development. Three dimensions are put forward in this study. They are sense of self-worth, mindset and emotion management. Furthermore, we discuss the approaches to promoting sense of self-worth, cultivating growth mindset and effectively managing emotions.
\end{abstract}

\section{Foreword}

In recent years, more and more attention has been drawn to personal development of university young teachers, especially to their psychological development. Zheng Xiaosheng ${ }^{[1]}$, Liu Xianmin ${ }^{[2]}$ and Zha Guoqing ${ }^{[3]}$, etc. analyzed young university teachers' status of psychological development and found that mental problems such as role stress, emotion disorder, and interpersonal relation disturbance, etc. exerted great influence on them. And they further discussed what universities could do to facilitate young teachers' psychological development. However, what could young university teachers do by themselves or which psychological factors they can improve to stimulate their development was rarely mentioned. Undoubtedly, favorable society and university policies contribute greatly to personal development of young university teachers. While generally external factors work through internal factors. Which psychological dimensions can immensely and effectively evoke their self-improvement and then generate an internal drive for overall development needs further discussion.

Liang Yamin put forward that the essence of teacher development lied in subjective development rather than that of a profession ${ }^{[4]}$. Wu Yujian also considered personal development of young university teachers very important and inadequately researched ${ }^{[5]}$. Based on development characteristics of young university teachers and status, this study proposes three psychological dimensions which are sense of self-worth, mindset and emotion management, and discussed approaches to improving sense of self-worth, developing growth mindset and skillfully managing emotions, attempting to provide reference for young university teachers.

\section{Significance and Status Analysis of Three Dimensions of Young University Teachers' Personal Development}

Psychological dimensions such as high sense of self-worth, growth mindset and effective emotion management act as powerful inner drive for personal development. However, some factors in environment or themselves lead to underdevelopment of the three aspects.

\section{Status of Young University Teachers' Sense of Self-worth}

Sense of self-worth is a psychological tendency to accept and affirm self-significance. Young university teachers with high sense of self-worth feel more secure and relatively safe from influence 
of outside assessment, disagreement and compliment. They have sufficient inner power to cope with crisis. Cultivation of young university teachers' high sense of self-worth can not only be of great significance to their sustainable development but also benefit students' positive sense of self-worth. However, Young university teachers with low sense of self-worth tend to underestimate themselves and more dependable on outside validation. A study found that young teachers with low sense of self-worth were inclined to punish students, pay more attention to students' defects and manipulate students. Therefore they have a negative effect on developing students' sense of self-worth.

Some young university teachers have low or unstable sense of self-worth. On the one hand, mostly under the age of 35, they lack an overall understanding of society, work environment and even themselves, which may lead to frustration and denial of self-worth. On the other hand, at the initial stage of their career, young university teachers are usually unskillful in teaching and researching, which is not conducive to boost their enthusiasm and confidence. Moreover, their low sense of self-worth may result from an unprivileged socioeconomic status. A research found that young university teachers are marginalized rather than centered in a university domain ${ }^{[7]}$.

\section{The Influence of Mindset on Young University Teachers}

A mindset can be seen as incident of a person's world view or philosophy of life. A mindset may be so firmly established that it creates a powerful incentive within these people or groups to continue to adopt or accept prior behaviors, choices, or tools ${ }^{[8]}$. Professor Carol Dweck from Stanford University divided mindset into fixed mindset and growth mindset. In her study, people with fixed mindset believe that their intelligence is unchangeable and therefore they are keen on proving their intelligence, focusing on results and neglecting the process of study. While people with growth mindset consider that their intelligence can be developed and cultivated and therefore they make great efforts to study and explore their potential. They are also apt to persist in the face of difficulties and eventually make greater achievements ${ }^{[9]}$.

The cultivation and application of growth mindset exert great influence on personal development of young university teachers and their guidance of students. Young university teachers with growth mindset can evaluate their abilities honestly and subjectively and make progress gradually by working hard. They view students in the light of development and highly affirm students' efforts. This value orientation conduct students to study hard, challenge themselves and sustain growth. Furthermore, a teacher with growth mindset can motivate their students to develop a growth mindset and make students benefit from it.

\section{Necessity of Emotion Management for Young University Teachers}

Emotion management skills refer to one's abilities to recognize and control emotions. People's emotions can be positive or negative. Positive emotions are pleasant and invigorate people's spirit to work efficiently. While negative emotions are usually unpleasant and depressing. They degrade people's enthusiasm for work and decrease efficiency.

In recent years, Domestic universities are launching widespread reform of evaluating systems. Some even implemented the "up or out" evaluation policy initiated and adopted by American research universities. Therefore, young university teachers are under great pressure in terms of teaching and researching in addition to marriage, house and kids raising problems. Some are under the influence of negative emotions such as anger, tension, anxiety, depression and helplessness. These negative emotions may discourage their enthusiasm for work and deteriorate their mental health conditions without guidance and management. In addition, improper release of negative emotions in class will create unsatisfactory performance and tarnished image of a young university teacher. 


\section{Approaches to Promoting Three Dimensions of Young University Teacher's Personal Development}

Personal development of young university teachers depend on the active role they play in the process. They can immensely upgrade their sense of self-worth, build up a growth mindset and effectively manage their negative emotions by adopting scientific methods. The three dimensions are interactive, interdependent and mutually promoted.

\section{Promotion of Sense of Self-worth}

High sense of self-worth is heavily dependable on a person's self-affirmation. Therefore, Young university teachers can achieve this by cognitive enhancement. Low sense of self-worth sometimes arise from incomprehensive understanding of themselves, others and incidents. They tend to underestimate and blame themselves for unfavorable conditions. Cognitive enhancement can motivate young university teachers to evaluate themselves, others and incidents more objectively, accept that defects and shortcomings are human nature. This stimulates young university teachers to eliminate self-doubt and self-denial and further construct a positive sense of self-worth.

Moreover, young university teachers with low sense of self-worth can also make progress by rebuilding an inner self-evaluation system ${ }^{[10]}$. The lack of an inner self-evaluation system causes young university teachers to be over-reliant on outside comment on them and dare not voice their true thoughts and feelings. Rebuilding an inner self-evaluation system means that young university teachers set out to attach importance to and respect their own perceptions and focus on their own value judgments rather than others'. When young university teachers fully accept themselves, they feel abundant sense of safety, neglect outside assessment and grow to be teachers with high sense of self-worth.

\section{Cultivation of Growth Mindset of Young University Teachers}

Growth mindset incorporates a set of beliefs towards intelligence, effort, setback and most significantly study. Therefore, young university teachers can develop a growth mindset by rooting growth beliefs and take growth actions.

(1) Value efforts highly. Young university teachers with growth mindset hold the opinion that their intelligence can be developed and promoted by studying continuously. They regard achieving success as a process during which they can accumulate knowledge and skills. For instance, young university teachers can not expect to greatly improve their ability of scientific research overnight. They need concentration and constant effort to generate valuable outcomes.

(2)Train developmental attribution towards setbacks. Young university teachers may suffer setbacks due to insufficient teaching experience and research paper writing skills such as failure in teaching competition or applying for research projects or rejection by a journal. They may feel depressed which is the first step to cope with setbacks. In the second and important step, they will try to identify possible causes, seek feedbacks to improvement and make more efforts. To them, failure means nothing but the request of more energy and efforts.

(3) Rise to challenges and difficulties. At the primary stage of their career, young university teachers will inevitably confront with challenges and difficulties. More often than not, challenges and difficulties cause unease, pain and sense of frustration. However, they are also incorporated with great opportunities to grow. Growth mindset advocates rising to challenges and difficulties by absorbing new knowledge, new ideas and employing more efficient methods. Young university teachers can make tremendous progress along with the overcome of challenges and difficulties.

\section{Master the Skills of Emotion Management}

Emotion Management of young university teachers emphasizes the proper expression and release of their negative emotions brought about by pressures of teaching, scientific research and personal lives. Therefore, negative emotions can no longer be a hindrance to their personal development.

It needs three steps to effectively manage emotions by young university teachers. First, be aware 
of and identify emotions. It is impossible to manage one's emotions without consciousness of the existence of negative emotions. After perception of negative emotions, they can define the emotions they perceived such as anger, disappointment, jealousy, sorrow, depression or boredom. Then accept the emotions. As is described by applied psychology, emotions are messengers of your inner self. If the messages are received, emotions will disappear. Therefore, acceptance of emotions is important. And then try to figure out what information the emotions attempt to send to you. Next, properly release emotions. Generally, if young university teachers' emotions are suppressed, the suppressed emotions may hurt themselves. And if they release them without proper control, negative emotions may hurt others. Therefore, the acceptable and effective means is appropriate expression of emotions. In expression of emotions, Young university teachers should take three aspects into consideration, which are emotions of their own, other's emotions and the situation ${ }^{[11]}$. In a suitable situation, young university teachers can convey their emotions with care for other's feelings and boost benign interaction with the world around them. Finally, they achieve their aim to effective emotion management.

\section{Conclusion}

As is analyzed above, personal and career development of young university teachers remain at a primary stage. They are open to new knowledge and ideas and therefore are equipped with great development potential. As subjective of personal development, only by bringing their initiative into full play can young university teachers take great advantages of the opportunities supplied by societies and universities. However, motivation of young university teachers' enthusiasm is inseparable from their psychological dimensions as high sense of self-worth, growth mindset and skillful emotion management. Therefore, this study put forward the three dimensions of young university teachers' personal development and analyzed how young university teachers could improve themselves in the three dimensions to provide reference for young university teachers' self-development.

\section{References}

[1] Zheng Xiaosheng, Psychological Status Analysis of Young University Teachers and Promotion Approaches [J]. Education Review, 2014 (12): 47-49.

[2] Liu Xianmin, Analysis of Source of Young University Teachers' Mental Stress and Mitigation Strategies [J]. Education Exploration, 2015 (10): 115-117.

[3] Zha Guoqing, Ma Xiting, Analysis of Young University Teachers' Mental Health and Promotion Strategies [J]. Management Observer, 2016 (13): 100-103.

[4] LiangYamin, Study on Objectivity alienation and Butterfly Transformation of Young University Teacher Development [J]. Chinese Adult Education, 2016 (22): 142-145.

[5] Wu Yujian, A Comparative Study of Chinese and American Young University Teachers' development [D]. Nanjing Normal University, Nanjing, 2014: 57.

[6] Chen Gaoshan, Huang Xiting, Sense of Self-worth of Young Teachers and Its Promotion Strategies [J]. Research in Higher Education, 1999 (1): 79-81.

[7] Han Meng, Zhang Guowei, A Study on Realistic Predicament and Reform Approaches of Young University Teacher Development [J]. Educational Science, 2017 (33): 43-49.

[8] https://en.wikipedia.org/wiki/Mindset

[9] Carol S. Dweck, Mindset-The New Psychology of Success [M]. Random House, New York, 2006: 15

[10] http://www.xinli001.com/info/100373773

[11] Huang Qituan, Price of Superciliousness [M]. Wenhui Publishing, Shanghai, 2017: 233. 